\title{
Robust Decentralized Adaptive Neural Control for a Class of Nonaffine Nonlinear Large-Scale Systems with Unknown Dead Zones
}

\author{
Huanqing Wang, ${ }^{1}$ Qi Zhou, ${ }^{2}$ Xuebo Yang, ${ }^{3}$ and Hamid Reza Karimi ${ }^{4}$ \\ ${ }^{1}$ School of Mathematics and Physics, Bohai University, Liaoning, Jinzhou 121000, China \\ ${ }^{2}$ College of Information Science and Technology, Bohai University, Liaoning, Jinzhou 121000, China \\ ${ }^{3}$ College of Engineering, Bohai University, Liaoning, Jinzhou 121000, China \\ ${ }^{4}$ Department of Engineering, Faculty of Engineering and Science, University of Agder, 4898 Grimstad, Norway \\ Correspondence should be addressed to Huanqing Wang; ndwhq@163.com
}

Received 6 January 2014; Accepted 11 February 2014; Published 19 March 2014

Academic Editor: Huaicheng Yan

Copyright (c) 2014 Huanqing Wang et al. This is an open access article distributed under the Creative Commons Attribution License, which permits unrestricted use, distribution, and reproduction in any medium, provided the original work is properly cited.

\begin{abstract}
The problem of robust decentralized adaptive neural stabilization control is investigated for a class of nonaffine nonlinear interconnected large-scale systems with unknown dead zones. In the controller design procedure, radical basis function (RBF) neural networks are applied to approximate packaged unknown nonlinearities and then an adaptive neural decentralized controller is systematically derived without requiring any information on the boundedness of dead zone parameters (slopes and break points). It is proven that the developed control scheme can ensure that all the signals in the closed-loop system are semiglobally uniformly ultimately bounded in the sense of mean square. Simulation study is provided to further demonstrate the effectiveness of the developed control scheme.
\end{abstract}

\section{Introduction}

During the past several decades, a large number of research results have been obtained on the problems of stability analysis and control design for nonlinear systems because of the extensive existence of nonlinearity in the practical systems. So far, there are many control methods proposed to control design of nonlinear systems, such as adaptive backstepping control [1-4], fault-tolerant control [5-9], $H_{\infty}$ control [10-16], and fuzzy control [17-21]. In the control of uncertain complex nonlinear systems, backstepping-based neural networks or fuzzy adaptive control technique is an efficient and practical strategy, and many interesting results have been obtained for a class of uncertain nonlinear strictfeedback systems; for example, see [22-37]. In addition, adaptive neural or fuzzy backstepping control approach can also be extended to control a class of nonaffine pure-feedback nonlinear systems representing a class of more general lower triangular systems, in which no affine appearance of the state variables can be used as virtual control signals. This makes it quite difficult and more meaningful to control the pure-feedback systems. By using small-gain theorem, in [38], an adaptive neural control scheme is presented for a class of completely nonaffine pure-feedback systems. Afterwards, some researchers further consider other types of pure-feedback nonlinear systems, such as pure-feedback systems with time-delay [39] and with dead-zone [40].

Large-scale system is considered as a dynamical system which is composed of some lower-order subsystems with interconnections and often exists in many practical applications such as electric power systems, computer network systems, and aerospace systems. Decentralized adaptive control of unknown nonlinear interconnected systems has attracted much research attention because this problem is important both theoretically and practically. Up to now, many interesting adaptive decentralized control approaches for large-scale nonlinear systems have been proposed in [41, 42] and the references therein. By combining backstepping technique together with adaptive neural or fuzzy control, many research papers have been published in [28-32] for 
affine nonlinear large-scale interconnected systems and in $[43,44]$ for nonaffine nonlinear large-scale systems. However, these researches have not considered the effect of the system input signal with dead zone. The existence of dead zone input nonlinearity severely degrades system performance. So, dead zone nonlinearity has to be considered when controller is designed in many industrial processes, such as valves, DC servo motors, and other devices. In [45], an adaptive robust control scheme is proposed for a class of nonlinear systems, in which the dead zone nonlinearity is expressed by a combination of a line and a disturbance-like term and its parameters are tuned by using adaptive technique. Then, some backstepping-based adaptive control schemes are developed for nonlinear systems with unknown dead zone input [46-50].

Based on the above observations, this paper focuses on the problem of adaptive neural decentralized control for a class of nonaffine large-scale nonlinear systems with unknown dead zones. The proposed adaptive neural controller guarantees that all the signals in the closed-loop systems remain semiglobally uniformly ultimately bounded in the sense of mean square, and the error signals eventually converge to small neighborhood around the origin. The main advantages of this research lie in that (i) the deadzone inverse as well as the prior knowledge of bounds of dead-zone parameters (slopes and break-points) is not required; (ii) only one adaptive parameter is involved in the developed controller for each subsystem. As a result, the computational burden is significantly alleviated. In this way, the proposed control law could be easily implemented in practical applications. Simulation results are provided to further illustrate the effectiveness of the proposed control approach.

The paper is organized as follows. Section 2 provides the problem formulation and preliminaries. The control design and analysis of state feedback controller are given in Section 3, following a simulation example in Section 4. Section 5 concludes this paper.

\section{Problem Formulation and Preliminaries}

Consider a class of pure-feedback nonlinear interconnected large-scale systems with dead zones and $N$ subsystems, and the $i$ th $(i=1,2, \ldots, N)$ subsystem is in the following form:

$$
\begin{gathered}
\dot{x}_{i, j}=f_{i, j}\left(\bar{x}_{i, j}, x_{i, j+1}\right)+\varphi_{i, j}(\bar{y}), \quad 1 \leq j \leq n_{i}-1, \\
\dot{x}_{i, n_{i}}=f_{i, n_{i}}\left(\bar{x}_{i, n_{i}}, u_{i}\right)+\varphi_{i, n_{i}}(\bar{y}), \\
y_{i}=x_{i, 1},
\end{gathered}
$$

where $\bar{x}_{i, j}=\left[x_{i, 1}, x_{i, 2}, \ldots, x_{i, j}\right]^{T}$ and $\bar{y}=\left[y_{1}, y_{2}, \ldots, y_{N}\right]^{T}$. $x_{i}=\left[x_{i, 1}, x_{i, 2}, \ldots, x_{i, n_{i}}\right]^{T} \in R^{n_{i}}$ and $y_{i} \in R$ are the state variables and the output of the $i$ th subsystem, respectively. $f_{i, j}(\cdot): R^{j+1} \rightarrow R,\left(j=1,2, \ldots, n_{i}\right)$ are unknown smooth nonlinear functions; $\varphi_{i, j}(\cdot): R^{N} \rightarrow R\left(j=1,2, \ldots, n_{i}\right)$ are unknown interconnections between the $i$ th subsystem and other subsystems, with $f_{i, j}(0)=\varphi_{i, j}(0)=0 ; u_{i} \in R$ is the output of an unknown dead zone and defined as

$$
u_{i}=D\left(v_{i}\right)= \begin{cases}g_{i r}\left(v_{i}\right), & v_{i} \geq b_{i r}, \\ 0, & b_{i l}<v_{i}<b_{i r} \\ g_{i l}\left(v_{i}\right), & v_{i} \leq b_{i l},\end{cases}
$$

where $b_{i l}<0$ and $b_{i r}>0$ are the unknown parameters and $v_{i}(t) \in R$ is the input of the dead zone.

For the unknown dead zone input, the following assumption is required.

Assumption 1 (see [40]). The functions, $g_{i l}\left(v_{i}\right)$ and $g_{i r}\left(v_{i}\right)$, are smooth and there exist unknown positive constants, $k_{i l 0}, k_{i l 1}, k_{i r 0}$, and $k_{i r 1}$, such that

$$
\begin{aligned}
& 0<k_{i l 0} \leq g_{i l}^{\prime}\left(v_{i}\right) \leq k_{i l 1}, \quad \forall v_{i} \in\left(-\infty, b_{i l}\right], \\
& 0<k_{i r 0} \leq g_{i r}^{\prime}\left(v_{i}\right) \leq k_{i r 1}, \quad \forall v_{i} \in\left[b_{i r},+\infty\right),
\end{aligned}
$$

and $\beta_{i 0} \leq \min \left\{k_{i l 0}, k_{i r 0}\right\}$ is an unknown positive constant, where $g_{i l}^{\prime}\left(v_{i}\right)=\left.\left(d g_{i l}(z) / d z\right)\right|_{z=v_{i}}$ and $g_{i r}^{\prime}\left(v_{i}\right)=$ $\left.\left(d g_{i r}(z) / d z\right)\right|_{z=v_{i}}$.

Remark 2. Assumption 1 is similar to the assumption in [40] where $\beta_{i 0}$ is a known constant. However, Assumption 1 does not require it to be known. So, Assumption 1 relaxes the limitation in [40].

Based on Assumption 1, the dead zone (2) can be rewritten as [40]

$$
u_{i}=D\left(v_{i}\right)=K_{i}^{T}(t) \Phi_{i}(t) v_{i}+d_{i}\left(v_{i}\right)
$$

where

$$
\begin{aligned}
& \Phi_{i}(t)=\left[\varphi_{i r}(t), \varphi_{i l}(t)\right]^{T}, \\
& \varphi_{i r}(t)= \begin{cases}1, & v_{i}(t)>b_{i l}, \\
0, & v_{i}(t) \leq b_{i l}\end{cases} \\
& \varphi_{i l}(t)= \begin{cases}1, & v_{i}(t)<b_{i r} \\
0, & v_{i}(t) \geq b_{i r}\end{cases} \\
& K_{i}(t)=\left[K_{i r}\left(v_{i}(t)\right), K_{i l}\left(v_{i}(t)\right)\right]^{T}, \\
& K_{i r}\left(v_{i}(t)\right)= \begin{cases}0, & v_{i}(t) \leq b_{i l}, \\
g_{i r}^{\prime}\left(\xi_{i r}\left(v_{i}(t)\right)\right), & b_{i l}<v_{i}(t)<+\infty,\end{cases} \\
& K_{i l}\left(v_{i}(t)\right)= \begin{cases}g_{i l}^{\prime}\left(\xi_{i l}\left(v_{i}(t)\right)\right), & -\infty<v_{i}(t)<b_{i r}, \\
0, & v_{i}(t) \geq b_{i r},\end{cases} \\
& d_{i}\left(v_{i}\right)= \begin{cases}-g_{i r}^{\prime}\left(\xi_{i r}\left(v_{i}\right)\right) b_{i r}, & v_{i} \geq b_{i r}, \\
-\left[g_{i l}^{\prime}\left(\xi_{i l}\left(v_{i}\right)\right)+g_{i r}^{\prime}\left(\xi_{i r}\left(v_{i}\right)\right)\right] v_{i}, & b_{i l}<v_{i}<b_{i r}, \\
-g_{i l}^{\prime}\left(\xi_{i l}\left(v_{i}\right)\right) b_{i l}, & v_{i} \leq b_{i l},\end{cases}
\end{aligned}
$$

where $\xi_{i l}\left(v_{i}\right) \in\left(v_{i}, b_{i l}\right)$, if $v_{i}<b_{i l} ; \xi_{i l}\left(v_{i}\right) \in\left(b_{i l}, v_{i}\right)$, if $b_{i l} \leq$ $v_{i}<b_{i r} ; \xi_{i r}\left(v_{i}\right) \in\left(b_{i r}, v_{i}\right)$, if $b_{i r}<v_{i} ; \xi_{i r}\left(v_{i}\right) \in\left(v_{i}, b_{i r}\right)$, and if $b_{i l}<v_{i} \leq b_{i r}$, and $\left|d_{i}\left(v_{i}\right)\right| \leq p_{i}^{*}, p_{i}^{*}$ is an unknown positive constant with $p^{*}=\left(k_{i r 1}+k_{i l 1}\right) \max \left\{b_{i r},-b_{i l}\right\}$. 
Remark 3. As shown in [40], there exist some other expressions for the case of linear dead zone outside the deadband, but (4) includes the most actual cases and $K_{i}^{T}(t) \Phi_{i}(t) \epsilon$ $\left[\beta_{i 0}, k_{i l 1}+k_{i r 1}\right] \subset(0,+\infty)$.

Based on mean value theorem [51], $f_{i, j}(\cdot)$ in $(1)$ can be described as

$$
\begin{gathered}
f_{i, j}\left(\bar{x}_{i, j}, x_{i, j+1}\right)=f_{i, j}\left(\bar{x}_{i, j}, x_{i, j+1}^{0}\right)+h_{\mu_{i, j}}\left(x_{i, j+1}-x_{i, j+1}^{0}\right), \\
f_{i, n_{i}}\left(\bar{x}_{i, n_{i}}, u_{i}\right)=f_{i, n_{i}}\left(\bar{x}_{i, n_{i}}, u_{i}^{0}\right)+h_{\mu_{i, n_{i}}}\left(u_{i}-u_{i}^{0}\right),
\end{gathered}
$$

where smooth function $f_{i, j}(\cdot)$ is explicitly analyzed between $f_{i, j}\left(\bar{x}_{i, j}, x_{i, j+1}\right)$ and $f_{i, j}\left(\bar{x}_{i, j}, x_{i, j+1}^{0}\right), h_{\mu_{i, j}}:=h_{i, j}\left(\bar{x}_{i, j}, x_{\mu_{i, j}}\right)=$ $\left.\left(\partial f_{i, j}\left(\bar{x}_{i, j}, x_{i, j+1}\right) / \partial x_{i, j+1}\right)\right|_{x_{i, j+1}=x_{\mu_{i, j}}}, x_{i, n_{i}+1}=u_{i}, x_{\mu_{i, j}}=$ $\mu_{i, j} x_{i, j+1}+\left(1-\mu_{i, j}\right) x_{i, j+1}^{0}, 0<\mu_{i, j}<1, i=1,2, \ldots, N, j=$ $1,2, \ldots, n_{i}$.

Next, by substituting (6) and (4) into (1), and choosing $x_{i, j+1}^{0}=0, u_{i}^{0}=0$, we obtain

$$
\begin{aligned}
& \dot{x}_{i, j}=h_{\mu_{i, j}} x_{i, j+1}+f_{i, j}\left(\bar{x}_{i, j}, 0\right)+\varphi_{i, j}(\bar{y}), \quad 1 \leq j \leq n_{i}-1, \\
& \dot{x}_{i, n_{i}}=h_{\mu_{i, n_{i}}} K_{i}^{T}(t) \Phi_{i}(t) v_{i}+h_{\mu_{i, n_{i}}} d_{i}\left(v_{i}\right)+f_{i, n_{i}}\left(\bar{x}_{i, n_{i}}, 0\right) \\
& +\varphi_{i, n_{i}}(\bar{y}) \\
& y_{i}=x_{i, 1}
\end{aligned}
$$

Assumption 4 (see [40]). The signs of $h_{\mu_{i, j}}, 1 \leq i \leq N, 1 \leq$ $j \leq n_{i}$, do not change, and there exist unknown constants $b_{m}$ and $c$ such that

$$
0<b_{m} \leq\left|h_{\mu_{i, j}}\right| \leq c<\infty
$$

Remark 5. Assumption 4 means that $h_{\mu_{i, j}}$ are strictly either positive or negative. Without loss of generality, it is assumed that $0<b_{m} \leq h_{\mu_{i, j}}$. In addition, by means of Assumptions 1 and 4 , it can be further supposed that

$$
\begin{gathered}
0<b \leq h_{\mu_{i, j}}, \quad 1 \leq i \leq N, \quad 1 \leq j \leq n_{i}-1, \\
0<b \leq h_{\mu_{i, n_{i}}} K_{i}^{T}(t) \Phi_{i}(t)
\end{gathered}
$$

where $b=\min \left\{b_{m}, b_{m} \beta_{i 0}\right\}$ is an unknown constant.

Assumption 6 (see [31]). For uncertain nonlinear functions $\varphi_{i, j}(\bar{y})$ in (1), there exist unknown smooth functions $\varphi_{i, j, l}\left(y_{l}\right)$ such that for $1 \leq i \leq N, 1 \leq j \leq n_{i}$,

$$
\left|\varphi_{i, j}(\bar{y})\right|^{2} \leq \sum_{l=1}^{N} \varphi_{i, j, l}^{2}\left(y_{l}\right)
$$

where $\varphi_{i, j, l}(0)=0, l=1,2, \ldots, N$.
Noting that $\varphi_{i, j, l}\left(y_{l}\right)$ in (10) is smooth function with $\varphi_{i, j, l}(0)=0$, so there exist unknown smooth functions $\bar{\varphi}_{i, j, l}\left(y_{l}\right)$ such that

$$
\left|\varphi_{i, j}(\bar{y})\right|^{2} \leq \sum_{l=1}^{N} y_{l}^{2} \bar{\varphi}_{i, j, l}^{2}\left(y_{l}\right)
$$

In what follows, RBF neural networks are applied to approximate any continuous function $f(X): R^{n} \rightarrow R$,

$$
f_{n n}(X)=W^{T} \phi(X),
$$

where $X \in \Omega_{Z} \subset R^{q}$ is the input vector with $q$ being the neural networks input dimension, weight vector $W=$ $\left[w_{1}, w_{2}, \ldots, w_{l}\right]^{T} \in R^{l}, l>1$ is the neural networks node number, and $\phi(X)=\left[\phi_{1}(X), \phi_{2}(X), \ldots, \phi_{l}(X)\right]^{T}$ means the basis function vector with $\phi_{i}(X)$ being chosen as the commonly used Gaussian function of the form

$$
\phi_{i}(X)=\exp \left[-\frac{\left(X-\mu_{i}\right)^{T}\left(X-\mu_{i}\right)}{\eta_{i}^{2}}\right], \quad i=1,2, \ldots, l,
$$

where $\mu_{i}=\left[\mu_{i 1}, \mu_{i 2}, \ldots, \mu_{i q}\right]^{T}$ is the center of the receptive field and $\eta_{i}$ is the width of the Gaussian function. In [52], it has been indicated that with sufficiently large node number $l$, the RBF neural networks (12) can approximate any continuous function $f(X)$ over a compact set $\Omega_{Z} \subset R^{q}$ to arbitrary any accuracy $\varepsilon>0$ as

$$
f(X)=W^{* T} \phi(X)+\delta(X), \quad \forall z \in \Omega_{z} \in R^{q},
$$

where $W^{*}$ is the ideal constant weight vector and defined as

$$
W^{*}:=\arg \min _{W \in \bar{R}^{l}}\left\{\sup _{Z \in \Omega_{Z}}\left|f(X)-W^{T} \phi(X)\right|\right\},
$$

and $\delta(X)$ denotes the approximation error and satisfies $|\delta(X)| \leq \varepsilon$.

Lemma 7 (see [53]). Consider the Gaussian RBF networks (12) and (2). Let $\rho:=(1 / 2) \min _{i \neq j}\left\|\mu_{i}-\mu_{j}\right\|$; then an upper bound of $\|\phi(X)\|$ is taken as

$$
\|\phi(X)\| \leq \sum_{k=0}^{\infty} 3 q(k+2)^{q-1} e^{-2 \rho^{2} k^{2} / \eta^{2}}:=s .
$$

It has been shown in [38] that the constant $s$ in Lemma 7 has a limited value and is independent of the variable $X$ and the dimension of neural weights $l$.

\section{Adaptive Neural Control Design}

In this section, an adaptive neural backstepping control scheme will be developed. During the controller design, for the $i$ th subsystem, RBF neural network $W_{i, j}^{T} \phi\left(X_{i, j}\right)$ will be utilized to approximate the packaged unknown function $F_{i, j}\left(X_{i, j}\right)$ at step $j$. Both the virtual control signals $\alpha_{i, j}\left(X_{i, j}\right)$ 
and adaption laws $\dot{\hat{\theta}}_{i}$ will be constructed in the following forms:

$$
\begin{aligned}
\alpha_{i, j}\left(X_{i, j}\right) & =-k_{i, j} z_{i, j}-\frac{1}{2 a_{i, j}^{2}} z_{i, j} \widehat{\theta}_{i} \phi_{i, j}^{T}\left(X_{i, j}\right) \phi_{i, j}\left(X_{i, j}\right), \\
\dot{\hat{\theta}}_{i} & =\sum_{j=1}^{n_{i}} \frac{\lambda_{i}}{2 a_{i, j}^{2}} z_{i, j}^{2} \phi_{i, j}^{T}\left(X_{i, j}\right) \phi_{i, j}\left(X_{i, j}\right)-\gamma_{i} \widehat{\theta}_{i}
\end{aligned}
$$

where $i=1,2, \ldots, N, j=1,2, \ldots, n_{i}, k_{i, j}, a_{i, j}, \lambda_{i}$, and $\gamma_{i}$ are positive design parameters, $X_{i, 1}=x_{i, 1}, X_{i, j}=\left[\bar{x}_{i, j}^{T}, \widehat{\theta}_{i}\right]^{T},(j=$ $\left.2, \ldots, n_{i}\right)$ with $\bar{x}_{i, j}=\left[x_{i, 1}, x_{i, 2}, \ldots, x_{i, j}\right]^{T}$, and $z_{i, j}$ satisfy the following coordinate transformation:

$$
z_{i, j}=x_{i, j}-\alpha_{i, j-1}
$$

with $\alpha_{i, 0}=0 . \widehat{\theta}_{i}$ is used to estimate an unknown constant $\theta_{i}$ which will be defined as

$$
\theta_{i}=\max \left\{\frac{1}{b}\left\|W_{i, j}\right\|^{2} ; j=1,2, \ldots, n_{i}\right\},
$$

where $b$ is specified in Remark 5 , and $\left\|W_{i, j}\right\|$ will be defined at the $j$ th step. Specially, $\alpha_{i, n_{i}}$ is the actual control input signal $u_{i}$.

For simplicity, in the following, the time variable $t$ and the state vector $\bar{x}_{i, j}$ will be omitted from the corresponding functions and let $\phi_{i, j}\left(X_{i, j}\right)=\phi_{i, j}$.

Step 1. Based on $z_{i, 1}=x_{i, 1}, z_{i, 2}=x_{i, 2}-\alpha_{i, 1}$, the error dynamic $z_{i, 1}$ satisfies

$$
\dot{z}_{i, 1}=h_{\mu_{i, 1}} z_{i, 2}+h_{\mu_{i, 1}} \alpha_{i, 1}+f_{i, j}\left(\bar{x}_{i, j}, 0\right)+\varphi_{i, 1}(\bar{y}) .
$$

Consider a Lyapunov function candidate as $V_{i, 1}=$ $(1 / 2) z_{i, 1}^{2}=(1 / 2) y_{i}^{2}$. Then, the time derivative of $V_{1}$ along (21) satisfies

$$
\dot{V}_{i, 1}=y_{i}\left(h_{\mu_{i, 1}} z_{i, 2}+h_{\mu_{i, 1}} \alpha_{i, 1}+f_{i, 1}\left(\bar{x}_{i, 1}, 0\right)+\varphi_{i, 1}(\bar{y})\right)
$$

By using (11) and the completion of squares, we get

$$
y_{i} \varphi_{i, 1}(\bar{y}) \leq \frac{1}{2} y_{i}^{2}+\frac{1}{2} \sum_{l=1}^{N} y_{l}^{2} \bar{\varphi}_{i, 1, l}^{2}\left(y_{l}\right)
$$

Further, (22) can be rewritten as

$$
\begin{aligned}
\dot{V}_{i, 1}= & y_{i}\left(h_{\mu_{i, 1}} z_{i, 2}+h_{\mu_{i, 1}} \alpha_{i, 1}+f_{i, 1}\left(\bar{x}_{i, 1}, 0\right)+\frac{1}{2} y_{i}\right) \\
& +\frac{1}{2} \sum_{l=1}^{N} y_{l}^{2} \bar{\varphi}_{i, 1, l}^{2}\left(y_{l}\right) .
\end{aligned}
$$

Step $j\left(2 \leq j \leq n_{i}-1\right)$. According to (19), one has

$$
\dot{z}_{i, j}=h_{\mu_{i, j}} z_{i, j+1}+h_{\mu_{i, j}} \alpha_{i, j}+f_{i, j}\left(\bar{x}_{i, j}, 0\right)+\varphi_{i, j}(\bar{y})-\dot{\alpha}_{i, j-1},
$$

where

$$
\dot{\alpha}_{i, j-1}=\sum_{k=1}^{j-1} \frac{\partial \alpha_{i, j-1}}{\partial x_{i, k}}\left(f_{i, k}\left(\bar{x}_{i, k+1}\right)+\varphi_{i, k}(\bar{y})\right)+\frac{\partial \alpha_{i, j-1}}{\partial \widehat{\hat{\theta}}_{i}}
$$

Take Lyapunov function $V_{i, j}=(1 / 2) z_{i, j}^{2}$; then we have

$$
\begin{aligned}
\dot{V}_{i, j}=z_{i, j}\left(g_{\mu_{i, j}} z_{i, j+1}+g_{\mu_{i, j}} \alpha_{i, j}+f_{i, j}\left(\bar{x}_{i, j}, 0\right)+\varphi_{i, j}(\bar{y})\right. & \\
& \left.-\sum_{k=1}^{j-1} \frac{\partial \alpha_{i, j-1}}{\partial x_{i, k}}\left(f_{i, k}\left(\bar{x}_{i, k+1}\right)+\varphi_{i, k}(\bar{y})\right)-\frac{\partial \alpha_{i, j-1}}{\partial \widehat{\hat{\theta}}_{i}}\right) .
\end{aligned}
$$

Following the same line as the procedures used in (23), one has

$$
\begin{gathered}
-z_{i, j} \sum_{k=1}^{j-1} \frac{\partial \alpha_{i, j-1}}{\partial x_{i, k}} \varphi_{i, k}(\bar{y}) \\
\leq \frac{1}{2} z_{i, j}^{2} \sum_{k=1}^{j-1}\left(\frac{\partial \alpha_{i, j-1}}{\partial x_{i, k}}\right)^{2}+\frac{1}{2} \sum_{k=1}^{j-1} \sum_{l=1}^{N} y_{l}^{2} \bar{\varphi}_{i, k, l}^{2}\left(y_{l}\right), \\
z_{i, j} \varphi_{i, j}(\bar{y}) \leq \frac{1}{2} z_{i, j}^{2}+\frac{1}{2} \sum_{l=1}^{N} y_{l}^{2} \bar{\varphi}_{i, j, l}^{2}\left(y_{l}\right) .
\end{gathered}
$$

It can be easily verified from (27) to (29) that

$$
\begin{aligned}
\dot{V}_{i, j} \leq z_{i, j}( & h_{\mu_{i, j}} z_{i, j+1}+h_{\mu_{i, j}} \alpha_{i, j}+f_{i, j}\left(\bar{x}_{i, j}, 0\right) \\
& -\sum_{k=1}^{j-1} \frac{\partial \alpha_{i, j-1}}{\partial x_{i, k}} f_{i, k}\left(\bar{x}_{i, k+1}\right)+\frac{1}{2} z_{i, j} \\
& \left.+\frac{1}{2} z_{i, j} \sum_{k=1}^{j-1}\left(\frac{\partial \alpha_{i, j-1}}{\partial x_{i, k}}\right)^{2}-\frac{\partial \alpha_{i, j-1}}{\partial \widehat{\theta}_{i}} \dot{\hat{\theta}}_{i}\right) \\
+ & \frac{1}{2} \sum_{k=1}^{j} \sum_{l=1}^{N} y_{l}^{2} \bar{\varphi}_{i, k, l}^{2}\left(y_{l}\right) .
\end{aligned}
$$

Step $n_{i}$. Similar to (25), the following equation can be obtained:

$$
\begin{aligned}
\dot{z}_{i, n_{i}}= & h_{\mu_{i, n_{i}}} K_{i}^{T}(t) \Phi_{i}(t) v_{i}+h_{\mu_{i, n_{i}}} d_{i}\left(v_{i}\right)+f_{i, n_{i}}\left(\bar{x}_{i, n_{i}}, 0\right) \\
& +\varphi_{i, n_{i}}(\bar{y})-\dot{\alpha}_{i, n_{i}-1},
\end{aligned}
$$

where $\dot{\alpha}_{i, n_{i}-1}$ is shown in (26) with $j=n_{i}$. Choose a Lyapunov function as

$$
V_{i, n_{i}}=\frac{1}{2} z_{i, n_{i}}^{2}+\frac{b}{2 \lambda_{i}} \widetilde{\theta}_{i}^{2}
$$

where $\widetilde{\theta}_{i}=\theta_{i}-\widehat{\theta}_{i}$ is the parameter error and $\lambda_{i}>0$ is a design parameter. 
Then, the following result holds.

$$
\begin{aligned}
& \dot{V}_{i, n_{i}}=z_{i, n_{i}}( h_{\mu_{i, n_{i}}} K_{i}^{T}(t) \Phi_{i}(t) v_{i}+h_{\mu_{i, n_{i}}} d_{i}\left(v_{i}\right) \\
&+f_{i, n_{i}}\left(\bar{x}_{i, n_{i}}, 0\right)+\varphi_{i, n_{i}}(\bar{y})-\frac{\partial \alpha_{i, n_{i}-1} \dot{\hat{\theta}}_{i}}{\partial \widehat{\theta}_{i}} \\
&\left.\quad-\sum_{k=1}^{n_{i}-1} \frac{\partial \alpha_{i, n_{i}-1}}{\partial x_{i, k}}\left(f_{i, k}\left(\bar{x}_{i, k+1}\right)+\varphi_{i, k}(\bar{y})\right)\right) \\
&-\frac{b}{\lambda_{i}} \widetilde{\theta}_{i} \dot{\hat{\theta}}_{i} .
\end{aligned}
$$

Repeating the same derivations as (28)-(30) produces

$$
\begin{gathered}
\dot{V}_{i, n_{i}} \leq z_{i, n_{i}}\left(h_{\mu_{i, n_{i}}} K_{i}^{T}(t) \Phi_{i}(t) v_{i}+h_{\mu_{i, n_{i}}} d_{i}\left(v_{i}\right)\right. \\
+f_{i, n_{i}}\left(\bar{x}_{i, n_{i}}, 0\right)-\sum_{k=1}^{n_{i}-1} \frac{\partial \alpha_{i, n_{i}-1}}{\partial x_{i, k}} f_{i, k}\left(\bar{x}_{i, k+1}\right) \\
\left.+\frac{1}{2} z_{i, n_{i}} \sum_{k=1}^{n_{i}-1}\left(\frac{\partial \alpha_{i, n_{i}-1}}{\partial x_{i, k}}\right)^{2}+\frac{1}{2} z_{i, n_{i}}-\frac{\partial \alpha_{i, n_{i}-1}}{\partial \widehat{\hat{\theta}}_{i}} \dot{\hat{\theta}}_{i}\right) \\
+\frac{1}{2} \sum_{k=1}^{n_{i}} \sum_{l=1}^{N} y_{l}^{2} \bar{\varphi}_{i, k, l}^{2}\left(y_{l}\right)-\frac{b}{\lambda_{i}} \tilde{\theta}_{i} \dot{\hat{\theta}}_{i} .
\end{gathered}
$$

Now, consider a Lyapunov function for the whole system as

$$
V=\sum_{i=1}^{N} \sum_{j=1}^{n_{i}} V_{i, j}=\sum_{i=1}^{N}\left(\frac{1}{2} y_{i}^{2}+\sum_{j=2}^{n_{i}} \frac{1}{2} z_{i, j}^{2}+\frac{b}{2 \lambda_{i}} \widetilde{\theta}_{i}^{2}\right) .
$$

Then, combining (24) together with (30) and (34), one has

$$
\begin{gathered}
\dot{V} \leq \sum_{i=1}^{N} y_{i}\left\{h_{\mu_{i, 1}} \alpha_{i, 1}+f_{i, 1}\left(\bar{x}_{i, 1}, 0\right)+\frac{1}{2} y_{i}\right. \\
\left.+\frac{1}{2} y_{i} \sum_{l=1}^{N} \sum_{s=1}^{n_{l}} \sum_{k=1}^{s} \bar{\varphi}_{l, k, i}^{2}\left(y_{i}\right)\right\} \\
+\sum_{i=1}^{N} \sum_{j=2}^{n_{i}-1} z_{i, j}\left\{h_{\mu_{i, j}} \alpha_{i, j}+h_{\mu_{i, j}} z_{i, j-1}\right. \\
+f_{i, j}\left(\bar{x}_{i, j}, 0\right)-\sum_{k=1}^{j-1} \frac{\partial \alpha_{i, j-1}}{\partial x_{i, k}} f_{i, k}\left(\bar{x}_{i, k+1}\right) \\
\left.+\frac{1}{2} z_{i, j}+\frac{1}{2} z_{i, j} \sum_{k=1}^{j-1}\left(\frac{\partial \alpha_{i, j-1}}{\partial x_{i, k}}\right)^{2}\right\}
\end{gathered}
$$

$$
\begin{aligned}
& +\sum_{i=1}^{N} z_{i, n_{i}}\left\{h_{\mu_{i, n_{i}}} K_{i}^{T}(t) \Phi_{i}(t) v_{i}+h_{\mu_{i, n_{i}}} d_{i}\left(v_{i}\right)\right. \\
& +f_{i, n_{i}}\left(\bar{x}_{i, n_{i}}, 0\right)-\sum_{k=1}^{n_{i}-1} \frac{\partial \alpha_{i, n_{i}-1}}{\partial x_{i, k}} f_{i, k}\left(\bar{x}_{i, k+1}\right) \\
& \left.+\frac{1}{2} z_{i, n_{i}} \sum_{k=1}^{n_{i}-1}\left(\frac{\partial \alpha_{i, n_{i}-1}}{\partial x_{i, k}}\right)^{2}+\frac{1}{2} z_{i, n_{i}}\right\} \\
& -\sum_{i=1}^{N} \sum_{j=2}^{n_{i}} z_{i, j} \frac{\partial \alpha_{i, j-1}}{\partial \widehat{\theta}_{i}} \dot{\hat{\theta}}_{i}-\sum_{i=1}^{N} \frac{b}{\lambda_{i}} \tilde{\theta}_{i} \dot{\hat{\theta}}_{i},
\end{aligned}
$$

where the fact of

$$
\begin{aligned}
\frac{1}{2} \sum_{i=1}^{N} \sum_{s=1}^{n_{i}} \sum_{k=1}^{s} \sum_{l=1}^{N} y_{l}^{2} \bar{\varphi}_{i, k, l}^{2}\left(y_{l}\right) \\
\quad=\frac{1}{2} \sum_{i=1}^{N} \sum_{l=1}^{N} \sum_{s=1}^{n_{l}} \sum_{k=1}^{s} y_{i}^{2} \bar{\varphi}_{l, k, i}^{2}\left(y_{i}\right)
\end{aligned}
$$

has been used in above inequality.

By using adaptive laws defined in (18) and rearranging sequence, we can obtain

$$
\begin{aligned}
& -\sum_{i=1}^{N} \sum_{j=2}^{n_{i}} z_{i, j} \frac{\partial \alpha_{i, j-1}}{\partial \widehat{\hat{\theta}}_{i}} \dot{\hat{\theta}}_{i} \\
& =-\sum_{i=1}^{N} \sum_{j=2}^{n_{i}} z_{i, j} \frac{\partial \alpha_{i, j-1}}{\partial \widehat{\theta}_{i}}\left(\sum_{k=1}^{n_{i}} \frac{\lambda_{i}}{2 a_{i, k}^{2}} z_{i, k}^{2} \phi_{i, k}^{T} \phi_{i, k}-\gamma_{i} \widehat{\theta}_{i}\right) \\
& =\sum_{i=1}^{N} \sum_{j=2}^{n_{i}} z_{i, j} \frac{\partial \alpha_{i, j-1}}{\partial \widehat{\theta}_{i}} \gamma_{i} \widehat{\theta}_{i} \\
& -\sum_{i=1}^{N} \sum_{j=2}^{n_{i}} z_{i, j} \frac{\partial \alpha_{i, j-1}}{\partial \widehat{\theta}_{i}} \sum_{k=1}^{j-1} \frac{\lambda_{i}}{2 a_{i, k}^{2}} z_{i, k}^{2} \phi_{i, k}^{T} \phi_{i, k} \\
& -\sum_{i=1}^{N} \sum_{j=2}^{n_{i}} z_{i, j} \frac{\partial \alpha_{i, j-1}}{\partial \widehat{\theta}_{i}} \sum_{k=j}^{n_{i}} \frac{\lambda_{i}}{2 a_{i, k}^{2}} z_{i, k}^{2} \phi_{i, k}^{T} \phi_{i, k} \\
& \leq \sum_{i=1}^{N} \sum_{j=2}^{n_{i}} z_{i, j} \frac{\partial \alpha_{i, j-1}}{\partial \widehat{\theta}_{i}} \gamma_{i} \widehat{\theta}_{i} \\
& -\sum_{i=1}^{N} \sum_{j=2}^{n_{i}} z_{i, j} \frac{\partial \alpha_{i, j-1}}{\partial \widehat{\theta}_{i}} \sum_{k=1}^{j-1} \frac{\lambda_{i}}{2 a_{i, k}^{2}} z_{i, k}^{2} \phi_{i, k}^{T} \phi_{i, k} \\
& +\sum_{i=1}^{N} \sum_{j=2}^{n_{i}} \frac{\lambda_{i}}{2 a_{i, j}^{2}} z_{i, j}^{2}\left(\sum_{k=2}^{j}\left|z_{i, k} \frac{\partial \alpha_{i, k-1}}{\partial \widehat{\theta}_{i}}\right|\right) .
\end{aligned}
$$

Taking $\left|d_{i}\left(v_{i}\right)\right| \leq p_{i}^{*}$ into account, one has

$$
z_{i, n_{i}} h_{\mu_{i, n_{i}}} d_{i}\left(v_{i}\right) \leq \frac{1}{2} z_{i, n_{i}}^{2} h_{\mu_{i, n_{i}}}^{2}+\frac{1}{2} p_{i}^{* 2}
$$


By substituting (38) and (39) into (36), it follows

$$
\begin{aligned}
\dot{V} \leq & \sum_{i=1}^{N} y_{i}\left(h_{\mu_{i, 1}} \alpha_{i, 1}+F_{i, 1}\left(X_{i, 1}\right)\right) \\
& +\sum_{i=1}^{N} \sum_{j=2}^{n_{i}-1} z_{i, j}\left(h_{\mu_{i, j}} \alpha_{i, j}+F_{i, j}\left(X_{i, j}\right)\right) \\
& +\sum_{i=1}^{N} z_{i, n_{i}}\left(h_{\mu_{i, n_{i}}} K_{i}^{T}(t) \Phi_{i}(t) v_{i}+F_{i, n_{i}}\left(X_{i, n_{i}}\right)\right) \\
& +\frac{1}{2} \sum_{i=1}^{N} p_{i}^{* 2}-\frac{1}{2} \sum_{i=1}^{N} \sum_{j=1}^{n_{i}} z_{i, j}^{2}-\sum_{i=1}^{N} \frac{b}{\lambda_{i}} \tilde{\theta}_{i} \dot{\hat{\theta}}_{i},
\end{aligned}
$$

where the packaged functions $F_{i, j}\left(X_{i, j}\right), i=1,2, \ldots, N$, are specified as

$$
\begin{aligned}
& F_{i, 1}\left(X_{i, 1}\right)=f_{i, 1}\left(\bar{x}_{i, 1}, 0\right)+y_{i}+\frac{1}{2} y_{i} \sum_{l=1}^{N} \sum_{s=1}^{n_{l}} \sum_{k=1}^{s} \bar{\varphi}_{l, k, i}^{2}\left(y_{i}\right), \\
& F_{i, j}\left(X_{i, j}\right)=h_{\mu_{i, j}} z_{i, j-1}+f_{i, j}\left(\bar{x}_{i, j}, 0\right)-\sum_{k=1}^{j-1} \frac{\partial \alpha_{i, j-1}}{\partial x_{i, k}} f_{i, k}\left(\bar{x}_{i, k+1}\right) \\
& +z_{i, j}+\frac{1}{2} z_{i, j} \sum_{k=1}^{j-1}\left(\frac{\partial \alpha_{i, j-1}}{\partial x_{i, k}}\right)^{2}+\frac{\partial \alpha_{i, j-1}}{\partial \widehat{\theta}_{i}} \gamma_{i} \widehat{\theta}_{i} \\
& -\frac{\partial \alpha_{i, j-1}}{\partial \widehat{\theta}_{i}} \sum_{k=1}^{j-1} \frac{\lambda_{i}}{2 a_{i, k}^{2}} z_{i, k}^{2} \phi_{i, k}^{T} \phi_{i, k} \\
& +\frac{\lambda_{i}}{2 a_{i, j}^{2}} z_{i, j}\left(\sum_{k=2}^{j}\left|z_{i, k} \frac{\partial \alpha_{i, k-1}}{\partial \widehat{\theta}_{i}}\right|\right) \\
& j=2, \ldots, n_{i}-1, \\
& F_{i, n_{i}}\left(X_{i, n_{i}}\right)=f_{i, n_{i}}\left(\bar{x}_{i, n_{i}}, 0\right)-\sum_{k=1}^{n_{i}-1} \frac{\partial \alpha_{i, n_{i}-1}}{\partial x_{i, k}} f_{i, k}\left(\bar{x}_{i, k+1}\right) \\
& +\frac{1}{2} z_{i, n_{i}} \sum_{k=1}^{n_{i}-1}\left(\frac{\partial \alpha_{i, n_{i}-1}}{\partial x_{i, k}}\right)^{2}+\frac{1}{2} z_{i, n_{i}} h_{\mu_{i, n_{i}}}^{2}+z_{i, n_{i}} \\
& +\frac{\partial \alpha_{i, n_{i}-1}}{\partial \widehat{\theta}_{i}} \gamma_{i} \widehat{\theta}_{i}-\frac{\partial \alpha_{i, n_{i}-1}}{\partial \widehat{\theta}_{i}} \sum_{k=1}^{n_{i}-1} \frac{\lambda_{i}}{2 a_{i, k}^{2}} z_{i, k}^{2} \phi_{i, k}^{T} \phi_{i, k} \\
& +\frac{\lambda_{i}}{2 a_{i, n_{i}}^{2}} z_{i, n_{i}}\left(\sum_{k=2}^{n_{i}}\left|z_{i, k} \frac{\partial \alpha_{i, k-1}}{\partial \widehat{\theta}_{i}}\right|\right) .
\end{aligned}
$$

Because $f_{i, j}, h_{\mu_{i, j}}$, and $\bar{\varphi}_{l, k, i}$ are unknown smooth functions, $F_{i, j}\left(X_{i, j}\right), i=1,2, \ldots, N, j=1,2, \ldots, n_{i}$ cannot be used to define the virtual control input $\alpha_{i, j}$ and the real controller $u_{i}$. Then, RBF neural network $W_{i, j}^{T} \phi_{i, j}\left(X_{i, j}\right)$ is utilized to model $F_{i, j}\left(X_{i, j}\right)$, such that for a given positive constant $\varepsilon_{i, j}$,

$$
F_{i, j}\left(X_{i, j}\right)=W_{i, j}^{T} \phi_{i, j}\left(X_{i, j}\right)+\delta_{i, j}\left(X_{i, j}\right),
$$

where $\delta_{i, j}\left(X_{i, j}\right)$ is approximation error and satisfies $\left|\delta_{i, j}\left(X_{i, j}\right)\right|<\varepsilon_{i, j}$. Subsequently, by Young's inequality, one has

$$
\begin{aligned}
& z_{i, j} F_{i, j}\left(X_{i, j}\right) \\
& =z_{i, j} \frac{W_{i, j}^{T}}{\left\|W_{i, j}\right\|} \phi_{i, j}\left\|W_{i, j}\right\|+z_{i, j} \delta_{i, j}\left(X_{i, j}\right) \\
& \leq \frac{b}{2 a_{i, j}^{2}} z_{i, j}^{2} \theta_{i} \phi_{i, j}^{T} \phi_{i, j}+\frac{1}{2} a_{i, j}^{2}+\frac{1}{2} z_{i, j}^{2}+\frac{1}{2} \varepsilon_{i, j}^{2},
\end{aligned}
$$

where $i=1,2, \ldots, N, j=1,2, \ldots, n_{i}$ and the unknown constant $\theta_{i}=\max \left\{(1 / b)\left\|W_{i, j}\right\|^{2} ; j=1,2, \ldots, n_{i}\right\}$.

Substituting (42) into (40) and using (43) produces

$$
\begin{aligned}
\dot{V} \leq & \sum_{i=1}^{N} z_{i, 1}\left(h_{\mu_{i, 1}} \alpha_{i, 1}+\frac{b}{2 a_{i, 1}^{2}} z_{i, 1} \theta_{i} \phi_{i, 1}^{T} \phi_{i, 1}\right) \\
& +\sum_{i=1}^{N} \sum_{j=2}^{n_{i}-1} z_{i, j}\left(h_{\mu_{i, j}} \alpha_{i, j}+\frac{b}{2 a_{i, j}^{2}} z_{i, j} \theta_{i} \phi_{i, j}^{T} \phi_{i, j}\right) \\
& +\sum_{i=1}^{N} z_{i, n_{i}}\left(h_{\mu_{i, n_{i}}} K_{i}^{T}(t) \Phi_{i}(t) v_{i}+\frac{b}{2 a_{i, n_{i}}^{2}} z_{i, n_{i}} \theta_{i} \phi_{i, n_{i}}^{T} \phi_{i, n_{i}}\right) \\
& +\frac{1}{2} \sum_{i=1}^{N} p_{i}^{* 2}+\sum_{i=1}^{N} \sum_{j=1}^{n_{i}}\left(\frac{1}{2} a_{i, j}^{2}+\frac{1}{2} \varepsilon_{i, j}^{2}\right)-\sum_{i=1}^{N} \frac{b}{\lambda_{i}} \widetilde{\theta}_{i} \dot{\hat{\theta}}_{i} .
\end{aligned}
$$

Now, construct the virtual control signals $\alpha_{i, j}=-k_{i, j} z_{i, j}-$ $\left(1 / 2 a_{i, j}^{2}\right) z_{i, j} \widehat{\theta}_{i} \phi_{i, j}^{T} \phi_{i, j}$, where $i=1,2, \ldots, N, j=1,2, \ldots, n_{i}$, and $\alpha_{i, n_{i}}=u_{i}$. Then, by using (9), we can obtain

$$
\begin{gathered}
z_{i, j} h_{\mu_{i, j}} \alpha_{i, j} \leq-k_{i, j} b z_{i, j}^{2}-\frac{b}{2 a_{i, j}^{2}} z_{i, j}^{2} \widehat{\theta}_{i} \phi_{i, j}^{T} \phi_{i, j}, \\
j=1, \ldots, n_{i}-1, \\
z_{i, n_{i}} h_{\mu_{i, n_{i}}} K_{i}^{T}(t) \Phi_{i}(t) v_{i} \leq-k_{i, n_{i}} b z_{i, n_{i}}^{2}-\frac{b}{2 a_{i, n_{i}}^{2}} z_{i, n_{i}}^{2} \widehat{\theta}_{i} \phi_{i, n_{i}}^{T} \phi_{i, n_{i}},
\end{gathered}
$$

where $i=1,2, \ldots, N$.

Substituting (45) into (44) and using adaptive laws $\dot{\theta}_{i}$ in (18) result in

$$
\begin{aligned}
\dot{V} \leq & \sum_{i=1}^{N} \sum_{j=1}^{n_{i}} z_{i, j}\left(-k_{i, j} b z_{i, j}+\frac{b}{2 a_{i, j}^{2}} z_{i, j} \widetilde{\theta}_{i} \phi_{i, j}^{T} \phi_{i, j}\right) \\
& +\sum_{i=1}^{N} \sum_{j=1}^{n_{i}}\left(\frac{1}{2} a_{i, j}^{2}+\frac{1}{2} \varepsilon_{i, j}^{2}\right)+\frac{1}{2} \sum_{i=1}^{N} p_{i}^{* 2} \\
& -\sum_{i=1}^{N} \frac{b}{\lambda_{i}} \widetilde{\theta}_{i}\left(\sum_{j=1}^{n_{i}} \frac{\lambda_{i}}{2 a_{i, j}^{2}} z_{i, j}^{2} \phi_{i, j}^{T} \phi_{i, j}-\gamma_{i} \widehat{\theta}_{i}\right)
\end{aligned}
$$




$$
\begin{aligned}
\leq & -\sum_{i=1}^{N} \sum_{j=1}^{n_{i}} k_{i, j} b z_{i, j}^{2}+\sum_{i=1}^{N} \frac{\gamma_{i} b}{\lambda_{i}} \widetilde{\theta}_{i} \widehat{\theta}_{i} \\
& +\sum_{i=1}^{N} \sum_{j=1}^{n_{i}}\left(\frac{1}{2} a_{i, j}^{2}+\frac{1}{2} \varepsilon_{i, j}^{2}\right)+\frac{1}{2} \sum_{i=1}^{N} p_{i}^{* 2} \\
\leq & -\sum_{i=1}^{N}\left(\sum_{j=1}^{n_{i}} k_{i, j} b z_{i, j}^{2}+\frac{\gamma_{i} b}{2 \lambda_{i}} \widetilde{\theta}_{i}^{2}\right) \\
& +\sum_{i=1}^{N} \sum_{j=1}^{n_{i}}\left(\frac{1}{2} a_{i, j}^{2}+\frac{1}{2} \varepsilon_{i, j}^{2}+\frac{\gamma_{i} b}{2 \lambda_{i}} \theta_{i}^{2}\right)+\frac{1}{2} \sum_{i=1}^{N} p_{i}^{* 2},
\end{aligned}
$$

where $\widetilde{\theta}_{i} \widehat{\theta}_{i} \leq-(1 / 2) \widetilde{\theta}_{i}^{2}+(1 / 2) \theta_{i}^{2}$ has been used in the above inequality.

Define

$$
\begin{gathered}
a_{0}=\min \left\{2 k_{i, j} b, \gamma_{i}, i=1,2, \ldots, N, j=1,2, \ldots, n_{i}\right\} \\
b_{0}=\sum_{i=1}^{N} \sum_{j=1}^{n_{i}}\left(\frac{1}{2} a_{i, j}^{2}+\frac{1}{2} \varepsilon_{i, j}^{2}+\frac{\gamma_{i} b}{2 \lambda_{i}} \theta_{i}^{2}\right)+\frac{1}{2} \sum_{i=1}^{N} p_{i}^{* 2}
\end{gathered}
$$

we have

$$
\dot{V} \leq-a_{0} V+b_{0}, \quad t \geq 0 .
$$

Furthermore, multiplying (48) by $e^{a_{0} t}$ yields

$$
\frac{d}{d t}\left(V e^{a_{0} t}\right) \leq b_{0} e^{a_{0} t}
$$

Integrating $(49)$ over $[0, t]$ gives

$$
0 \leq V(t) \leq \frac{b_{0}}{a_{0}}+\left(V(0)-\frac{b_{0}}{a_{0}}\right) e^{-a_{0} t},
$$

which implies that

$$
V(t) \leq V(0)+\frac{b_{0}}{a_{0}}, \quad \forall t>0,
$$

which means that all the signals in the closed-loop system are semiglobally uniformly ultimately bounded.

Furthermore, it is easily obtained that

$$
V(t) \leq \frac{b_{0}}{a_{0}}, \quad t \longrightarrow+\infty .
$$

Therefore, based on the definition of $V$ in (50), the error signals $z_{i, j}$ and $\widetilde{\theta}_{i}$ eventually converge to the compact set $\Omega_{s}$, which is specified as

$$
\begin{gathered}
\Omega_{s}=\left\{z_{i, j}, \tilde{\theta}_{i}|| z_{i, j}\left|\leq \sqrt{2 \frac{b_{0}}{a_{0}}},\right| \widetilde{\theta}_{i} \mid \leq \sqrt{\frac{2 \lambda_{i}}{b} \frac{b_{0}}{a_{0}}},\right. \\
\left.1 \leq i \leq N, 1 \leq j \leq n_{i}\right\} .
\end{gathered}
$$

At the present stage, adaptive decentralized control procedure has been completed via backstepping technique. The above design procedures and stable analysis are summarized in the following theorem.

Theorem 8. Under Assumptions 1-6, consider the closedloop nonlinear system consisted of (1), unknown dead zone nonlinearities (2), controller (17), and adaptive law (18). Under the action of controller (17), for any initial conditions $\left[z_{i}^{T}(0), \hat{\theta}_{i}(0)\right]^{T} \in \Omega_{0}$ (where $\Omega_{0}$ is an appropriately chosen compact set), all the signals in the closed-loop system are semiglobally uniformly ultimately bounded in the sense of mean square, and the error signals $z_{i, j}$ and $\tilde{\theta}_{i}$ eventually converge to the compact set $\Omega_{s}$ in (53).

\section{Simulation Example}

In this section, in order to illustrate the effectiveness of the proposed control scheme, consider the nonaffine interconnected nonlinear system with dead zones as

$$
\begin{gathered}
\dot{x}_{1,1}=\left(1+x_{1,1}^{2}\right) x_{1,2}+x_{1,2}^{3}+y_{2} \ln \left(1+y_{1}^{2}\right), \\
\dot{x}_{1,2}=\left(5+\sin \left(x_{1,1} x_{1,2}\right)\right) u_{1}+0.2 u_{1}^{5}+\sin \left(y_{1}^{2}\right) y_{2}, \\
y_{1}=x_{1,1}, \\
\dot{x}_{2,1}=\left(3+\sin \left(x_{2,1}\right)\right) x_{2,2}+0.3 x_{2,2}^{5}+y_{1} \cos \left(y_{2}^{2}\right), \\
\dot{x}_{2,2}=\left(2+x_{2,1}^{2}\right) u_{2}+\frac{1}{5} \sin \left(u_{2}\right)+y_{2}^{3} y_{1}, \\
y_{2}=x_{2,1},
\end{gathered}
$$

where $u_{i}=D\left(v_{i}\right)$ is defined as

$$
\begin{aligned}
& u_{1}=D\left(v_{1}\right)= \begin{cases}1.5\left(v_{1}-2.5\right), & v_{1} \geq 2.5, \\
0, & -1<v_{1}<2.5, \\
0.5\left(v_{1}+1\right), & v_{1} \leq-1,\end{cases} \\
& u_{2}=D\left(v_{2}\right)= \begin{cases}0.8\left(v_{2}-2\right), & v_{2} \geq 2, \\
0, & -1.8<v_{2}<2, \\
1.2\left(v_{2}+1.8\right), & v_{2} \leq-1.8,\end{cases}
\end{aligned}
$$

where $x_{1,1}, x_{1,2}, x_{2,1}$, and $x_{2,2}$ are the state variables and $y_{i}$ is the system output. $u_{i}$ and $v_{i}$ are the output and input of the dead zone nonlinearity, respectively. It is obvious that the system is of nonaffine structure and satisfies Assumptions 1, 4, and 6. By using Theorem 8, the virtual control law, the actual controller, and the adaptive laws are constructed as

$$
\begin{gathered}
\alpha_{i, 1}=-k_{i, 1} z_{i, 1}-\frac{1}{2 a_{i, 1}^{2}} z_{i, 1} \widehat{\theta}_{i} \phi_{i, 1}^{T} \phi_{i, 1}, \\
u_{i}=-k_{i, 2} z_{i, 2}-\frac{1}{2 a_{i, 2}^{2}} z_{i, 2} \widehat{\theta}_{i} \phi_{i, 2}^{T} \phi_{i, 2}, \\
\dot{\widehat{\theta}}_{i}=\sum_{j=1}^{2} \frac{\lambda_{i}}{2 a_{i, j}^{2}} z_{i, j}^{2} \phi_{i, j}^{T} \phi_{i, j}-\gamma_{i} \widehat{\theta}_{i},
\end{gathered}
$$




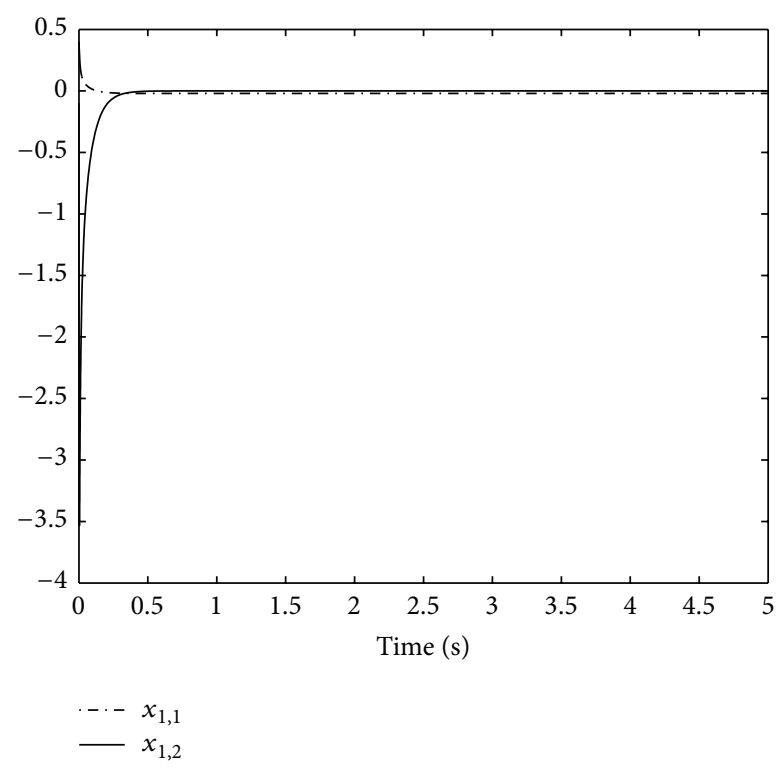

Figure 1: State variables $x_{1,1}$ and $x_{1,2}$.

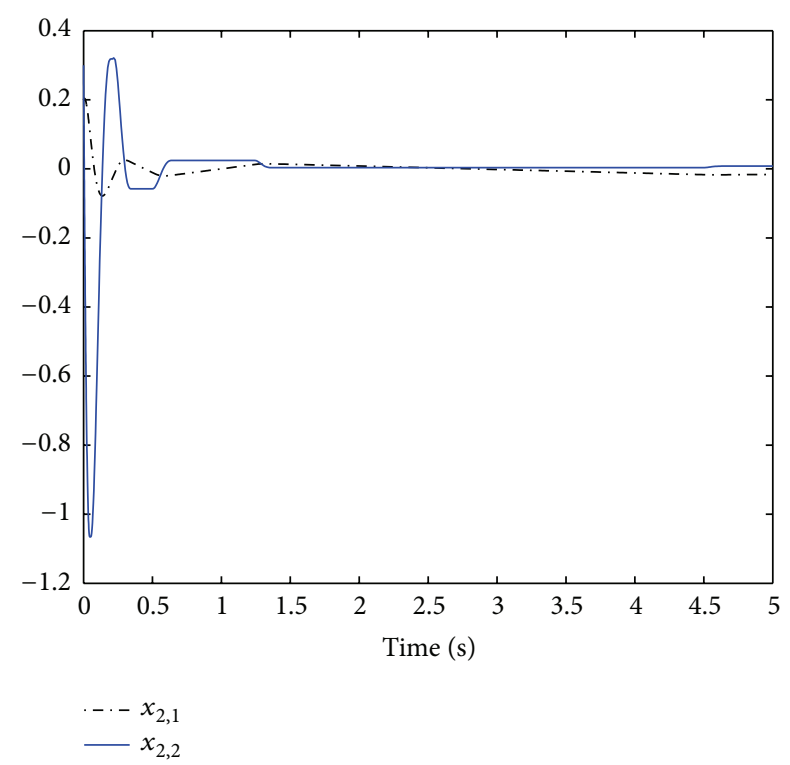

FIgURE 2: State variables $x_{2,1}$ and $x_{2,2}$.

where $z_{i, 1}=x_{i, 1}, z_{i, 2}=x_{i, 2}-\alpha_{i, 1}$, and $i=1,2$. The simulation is run under the initial conditions $\left[x_{1,1}(0)\right.$, $\left.x_{1,2}(0), x_{2,1}(0), x_{2,2}(0)\right]^{T}=[0.4,-0.1,0.2,0.3]^{T}$, and $\left[\hat{\theta}_{1}(0)\right.$, $\left.\widehat{\theta}_{2}(0)\right]^{T}=[0.1,0.2]^{T}$. In the simulation, design parameters are taken as follows: $k_{1,1}=k_{1,2}=k_{2,1}=k_{2,2}=11, a_{1,1}=$ $a_{1,2}=a_{2,1}=a_{2,2}=3, \gamma_{1}=\gamma_{2}=1$, and $\lambda_{1}=\lambda_{2}=2$.

The simulation results are indicated by Figures 1-4. Figure 1 shows the state variables $x_{1,1}$ and $x_{1,2}$ of the first subsystems. Figure 2 shows the second subsystems state variables $x_{2,1}$ and $x_{2,2}$. Figure 3 displays the control signals $v_{1}$ and $v_{2}$, and Figure 4 shows the response curve of the adaptive parameters $\widehat{\theta}_{1}$ and $\hat{\theta}_{2}$. Apparently, simulation results show

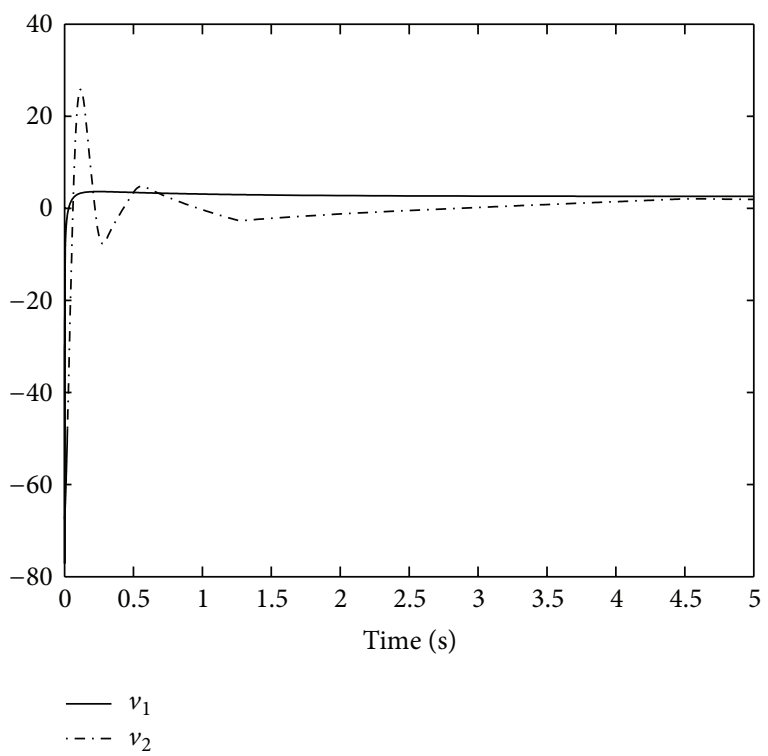

FIgURE 3: The control signals $v_{1}$ and $v_{2}$.

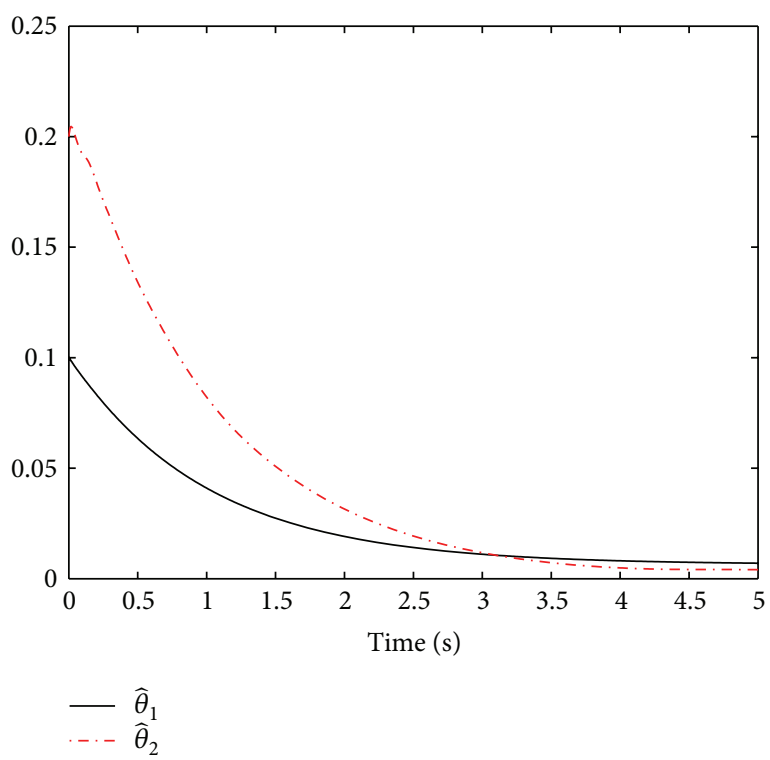

Figure 4: Adaptive laws $\widehat{\theta}_{1}$ and $\widehat{\theta}_{2}$.

that good convergence performances are achieved and all the signals in the closed-loop system are bounded.

\section{Conclusion}

In this paper, a robust decentralized adaptive neural control approach has been developed for a class of large-scale nonaffine nonlinear systems with dead zones. The proposed decentralized controller can guarantee that all the signals in the closed-loop systems are semiglobally uniformly ultimately bounded in the sense of mean square, and the error signals eventually converge to small neighborhood around the origin. The main advantage of the proposed controller 
is that the prior knowledge of bounds of dead zone slopes is not required. In addition, only one adaptive parameter needs to be updated online for each $n$-order subsystem. In this way, the computational burden is significantly alleviated. Simulation results have been provided to further illustrate the effectiveness of our results.

There are some problems remaining to be considered, for example, how to generalize the result in this paper to stochastic pure-feedback nonlinear systems with dead zones and how to design an output-feedback adaptive neural controller for original system (1).

\section{Conflict of Interests}

The authors declare that there is no conflict of interests regarding the publication of this paper.

\section{Acknowledgments}

This work is partially supported by the Natural Science Foundation of China (61304002 and 61304003), the Program for New Century Excellent Talents in University (NECT-130696), the Program for Liaoning Innovative Research Team in University under Grant LT2013023, the Program for Liaoning Excellent Talents in University under Grant LR2013053, the Polish-Norwegian Research Programme operated by the National Centre for Research and 24 Development under the Norwegian Financial Mechanism 2009-2014 in the frame of Project Contract no. Pol-Nor/200957/47/2013, and the Education Department of Liaoning Province under the General Project Research Grant no. L2013424.

\section{References}

[1] M. Krstić, I. Kanellakopoulos, and P. V. Kokotovic, Nonlinear and Adaptive Control Design, Wiley, New York, NY, USA, 1995.

[2] Z. Wu, M. Cui, P. Shi, and H. R. Karimi, "Stability of stochastic nonlinear systems with state-dependent switching," IEEE Transactions on Automatic Control, vol. 58, no. 8, pp. 1904-1918, 2013.

[3] L. Liu and X.-J. Xie, "Output-feedback stabilization for stochastic high-order nonlinear systems with time-varying delay," Automatica, vol. 47, no. 12, pp. 2772-2779, 2011.

[4] L. Liu, N. Duan, and X.-J. Xie, "Output-feedback stabilization for stochastic high-order nonlinear systems with a ratio of odd integers power," Acta Automatica Sinica, vol. 36, no. 6, pp. 858864, 2010.

[5] S. Yin, S. Ding, and H. Luo, "Real-time implementation of fault tolerant control system with performance optimization," IEEE Transactions on Industrial Electronics, vol. 61, no. 5, pp. 24022411, 2014.

[6] S. Yin, S. Ding, A. Haghani, H. Hao, and P. Zhang, "A comparison study of basic data-driven fault diagnosis and process monitoring methods on the benchmark Tennessee Eastman process," Journal of Process Control, vol. 22, no. 9, pp. 1567-1581, 2012.

[7] M. Liu, X. Cao, and P. Shi, "Fault estimation and tolerant control for fuzzy stochastic systems," IEEE Transactions on Fuzzy Systems, vol. 21, no. 2, pp. 221-229, 2013.
[8] M. Liu, X. Cao, and P. Shi, "Fuzzy-model-based fault tolerant design for nonlinear stochastic systems against simultaneous sensor and actuator faults," IEEE Transactions on Fuzzy Systems, vol. 21, no. 5, pp. 789-799, 2013.

[9] X. D. Zhao, L. X. Zhang, P. Shi, and H. R. Karimi, "Robust control of continuous-time systems with state-dependent uncertainties and its application to electronic circuits," IEEE Transactions on Industrial Electronics, vol. 61, no. 8, pp. 4161-4170, 2014.

[10] H. R. Karimi, "Robust delay-dependent $H_{\infty}$ control of uncertain time-delay systems with mixed neutral, discrete, and distributed time-delays and Markovian switching parameters," IEEE Transactions on Circuits and Systems, vol. 58, no. 8, pp. 1910-1923, 2011.

[11] H. Y. Li, X. J. Jing, and H. R. Karimi, "Output-feedback based $\mathrm{H}$-infinity control for active suspension systems with control delay," IEEE Transactions on Industrial Electronics, vol. 61, no. 1, pp. 436-446, 2014.

[12] H. Gao, T. Chen, and J. Lam, "A new delay system approach to network-based control," Automatica, vol. 44, no. 1, pp. 39-52, 2008

[13] L. X. Zhang, H. J. Gao, and O. Kaynak, "Network-induced constraints in networked control systems-a survey," IEEE Transactions on Industrial Informatics, vol. 9, no. 1, pp. 403-416, 2013.

[14] H. Yan, Z. Su, H. Zhang, and F. Yang, "Observer-based $H_{\infty}$ control for discrete-time stochastic systems with quantisation and random communication delays," IET Control Theory of Applications, vol. 7, no. 3, pp. 372-379, 2013.

[15] H. Yan, H. Shi, H. Zhang, and F. Yang, "Quantized $H_{\infty}$ control for networked systems with communication constraints," Asian Journal of Control, vol. 15, no. 5, pp. 1468-1476, 2013.

[16] H. Zhang, Q. Chen, H. Yan, and J. Liu, "Robust $H_{\infty}$ filtering for switched stochastic system with missing measurements," IEEE Transactions on Signal Processing, vol. 57, no. 9, pp. 3466-3474, 2009.

[17] H. Zhang, H. Yan, T. Liu, and Q. Chen, "Fuzzy controller design for nonlinear impulsive fuzzy systems with time delay," IEEE Transactions on Fuzzy Systems, vol. 19, no. 5, pp. 844-856, 2011.

[18] H. Zhang, H. Yan, F. Yang, and Q. Chen, "Quantized control design for impulsive fuzzy networked systems," IEEE Transactions on Fuzzy Systems, vol. 19, no. 6, pp. 1153-1162, 2011.

[19] H. Y. Li, J. Y. Yu, C. Hilton, and H. H. Liu, "Adaptive sliding mode control for nonlinear active suspension vehicle systems using T-S fuzzy approach," IEEE Transactions on Industrial Electronics, vol. 60, no. 8, pp. 3328-3338, 2013.

[20] X. D. Zhao, L. X. Zhang, P. Shi, and H. R. Karimi, "Novel stability criteria for T-S fuzzy systems," IEEE Transactions on Fuzzy Systems, vol. 21, no. 6, pp. 1-11, 2013.

[21] X. Su, P. Shi, L. Wu, and Y. Song, "A novel approach to filter design for T-S fuzzy discretetime systems with time-varying delay," IEEE Transactions on Fuzzy Systems, vol. 20, no. 6, pp. 1114-1129, 2012.

[22] H. Q. Wang, B. Chen, X. P. Liu, K. F. Liu, and C. Lin, "Robust adaptive fuzzy tracking control for pure-feedback stochastic nonlinear systems with input constraints," IEEE Transaction on Cybernetics, vol. 43, no. 6, pp. 2093-2104, 2013.

[23] S. Bououden, M. Chadli, F. Allouani, and S. Filali, "A new approach for fuzzy predictive adaptive controller design using particle swarm optimization algorithm," International Journal of Innovative Computing, Information and Control, vol. 9, no. 9, pp. 3741-3758, 2013. 
[24] S. Sefriti, J. Boumhidi, M. Benyakhlef, and I. Boumhidi, "Adaptive decentralized sliding mode neural network control of a class of nonlinear interconnected systems," International Journal of Innovative Computing, Information and Control, vol. 9, no. 7, pp. 2941-2947, 2013.

[25] Y.-J. Liu, W. Wang, S.-C. Tong, and Y.-S. Liu, "Robust adaptive tracking control for nonlinear systems based on bounds of fuzzy approximation parameters," IEEE Transactions on Systems, Man, and Cybernetics A, vol. 40, no. 1, pp. 170-184, 2010.

[26] H. Q. Wang, B. Chen, and C. Lin, "Approximation-based adaptive fuzzy control for a class of non-strict-feedback stochastic nonlinear systems," Science China Information Sciences, 2013.

[27] T. Shaocheng, C. Bin, and W. Yongfu, "Fuzzy adaptive output feedback control for MIMO nonlinear systems," Fuzzy Sets and Systems, vol. 156, no. 2, pp. 285-299, 2005.

[28] W. Chen and J. Li, "Decentralized output-feedback neural control for systems with unknown interconnections," IEEE Transactions on Systems, Man, and Cybernetics B, vol. 38, no. 1, pp. 258-266, 2008.

[29] S. Tong, C. Liu, and Y. Li, "Fuzzy-adaptive decentralized output-feedback control for large-scale nonlinear systems with dynamical uncertainties," IEEE Transactions on Fuzzy Systems, vol. 18, no. 5, pp. 845-861, 2010.

[30] S. C. Tong, Y. M. Li, and H.-G. Zhang, "Adaptive neural network decentralized backstepping output-feedback control for nonlinear large-scale systems with time delays," IEEE Transactions on Neural Networks, vol. 22, no. 7, pp. 1073-1086, 2011.

[31] J. Li, W. Chen, and J.-M. Li, "Adaptive NN output-feedback decentralized stabilization for a class of large-scale stochastic nonlinear strict-feedback systems," International Journal of Robust and Nonlinear Control, vol. 21, no. 4, pp. 452-472, 2011.

[32] H. Q. Wang, B. Chen, and C. Lin, "Adaptive fuzzy decentralized control for a class of large-scale stochastic nonlinear strictfeedback systems," Neurocomputing, vol. 103, pp. 155-163, 2013.

[33] H. Q. Wang, B. Chen, K. F. Liu, X. P. Liu, and C. Lin, "Adaptive neural tracking control for a class of non-strictfeedback stochastic nonlinear systems with unknown backlashlike hysteresis," IEEE Transactions on Neural Networks and Learning Systems, 2013.

[34] H. Y. Li, X. J. Jing, H. K. Lam, and P. Shi, "Fuzzy sampleddata control for uncertain vehicle suspension systems," IEEE Transactions on Cybernetics, 2013.

[35] T.-S. Li, D. Wang, G. Feng, and S.-C. Tong, "A DSC approach to robust adaptive nn tracking control for strict-feedback nonlinear systems," IEEE Transactions on Systems, Man, and Cybernetics B, vol. 40, no. 3, pp. 915-927, 2010.

[36] M. Chen, S. S. Ge, and B. V. E. How, "Robust adaptive neural network control for a class of uncertain MIMO nonlinear systems with input nonlinearities," IEEE Transactions on Neural Networks, vol. 21, no. 5, pp. 796-812, 2010.

[37] B. Chen, X. Liu, K. Liu, and C. Lin, "Direct adaptive fuzzy control of nonlinear strict-feedback systems," Automatica, vol. 45 , no. 6, pp. 1530-1535, 2009.

[38] C. Wang, D. J. Hill, S. S. Ge, and G. Chen, "An ISS-modular approach for adaptive neural control of pure-feedback systems," Automatica, vol. 42, no. 5, pp. 723-731, 2006.

[39] M. Wang, S. S. Ge, and K.-S. Hong, "Approximation-based adaptive tracking control of pure-feedback nonlinear systems with multiple unknown time-varying delays," IEEE Transactions on Neural Networks, vol. 21, no. 11, pp. 1804-1816, 2010.
[40] T. P. Zhang and S. S. Ge, "Adaptive dynamic surface control of nonlinear systems with unknown dead zone in pure feedback form," Automatica, vol. 44, no. 7, pp. 1895-1903, 2008.

[41] Z. Huaguang and L. Cai, "Decentralized nonlinear adaptive control of an HVAC system," IEEE Transactions on Systems, Man and Cybernetics C, vol. 32, no. 4, pp. 493-498, 2002.

[42] J. Zhou and C. Wen, "Decentralized backstepping adaptive output tracking of interconnected nonlinear systems," IEEE Transactions on Automatic Control, vol. 53, no. 10, pp. 23782384, 2008.

[43] B. Karimi and M. B. Menhaj, "Non-affine nonlinear adaptive control of decentralized large-scale systems using neural networks," Information Sciences, vol. 180, no. 17, pp. 3335-3347, 2010.

[44] Z.-Z. Mao and X.-S. Xiao, "Decentralized adaptive tracking control of nonaffine nonlinear large-scale systems with time delays," Information Sciences, vol. 181, no. 23, pp. 5291-5303, 2011.

[45] X.-S. Wang, C.-Y. Su, and H. Hong, "Robust adaptive control of a class of nonlinear systems with unknown dead-zone," Automatica, vol. 40, no. 3, pp. 407-413, 2004.

[46] J. Zhou, C. Wen, and Y. Zhang, "Adaptive output control of nonlinear systems with uncertain dead-zone nonlinearity," IEEE Transactions on Automatic Control, vol. 51, no. 3, pp. 504511, 2006.

[47] S. Tong and Y. Li, "Adaptive fuzzy output feedback tracking backstepping control of strict-feedback nonlinear systems with unknown dead zones," IEEE Transactions on Fuzzy Systems, vol. 20, no. 1, pp. 168-180, 2012.

[48] H. Wang, B. Chen, and C. Lin, "Direct adaptive neural tracking control for a class of stochastic pure-feedback nonlinear systems with unknown dead-zone," International Journal of Adaptive Control and Signal Processing, vol. 27, no. 4, pp. 302-322, 2013.

[49] H. Q. Wang, B. Chen, and C. Lin, "Adaptive neural tracking control for a class of stochastic nonlinear systems with unknown dead-zone," International Journal of Innovative Computing, Information and Control, vol. 9, no. 8, pp. 3257-3270, 2013.

[50] Y. M. Li and S. C. Tong, "Adaptive fuzzy output-feedback control of pure-feedback uncertain nonlinear systems with unknown dead-zone," IEEE Transactions on Fuzzy Systems, 2013.

[51] T. M. Apostol, Mathematical Analysis, Addison-Wesley, Reading, Mass, USA, 1963.

[52] R. M. Sanner and J.-J. E. Slotine, "Gaussian networks for direct adaptive control," IEEE Transactions on Neural Networks, vol. 3, no. 6, pp. 837-863, 1992.

[53] A. J. Kurdila, F. J. Narcowich, and J. D. Ward, "Persistency of excitation in identification using radial basis function approximants," SIAM Journal on Control and Optimization, vol. 33, no. 2, pp. 625-642, 1995. 


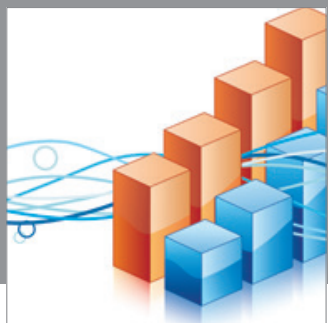

Advances in

Operations Research

mansans

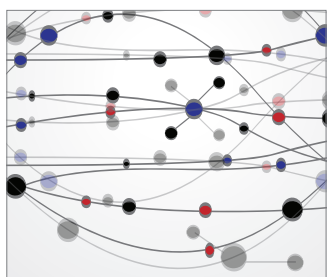

The Scientific World Journal
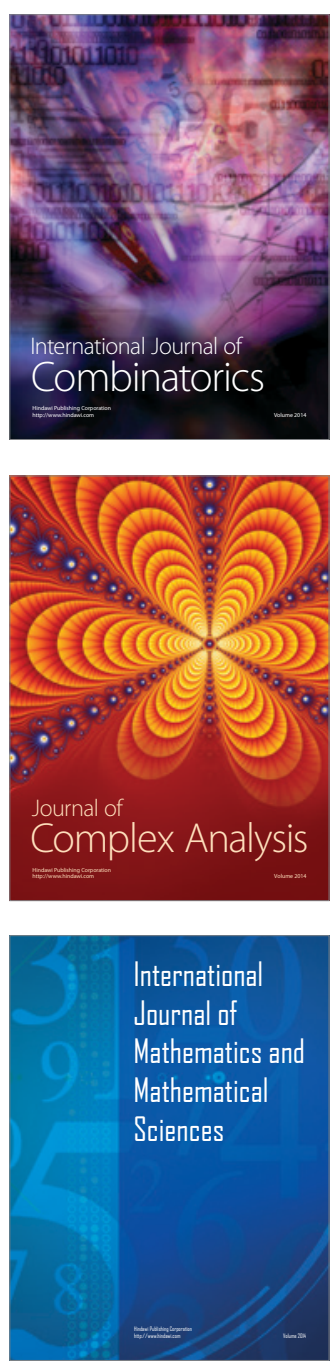
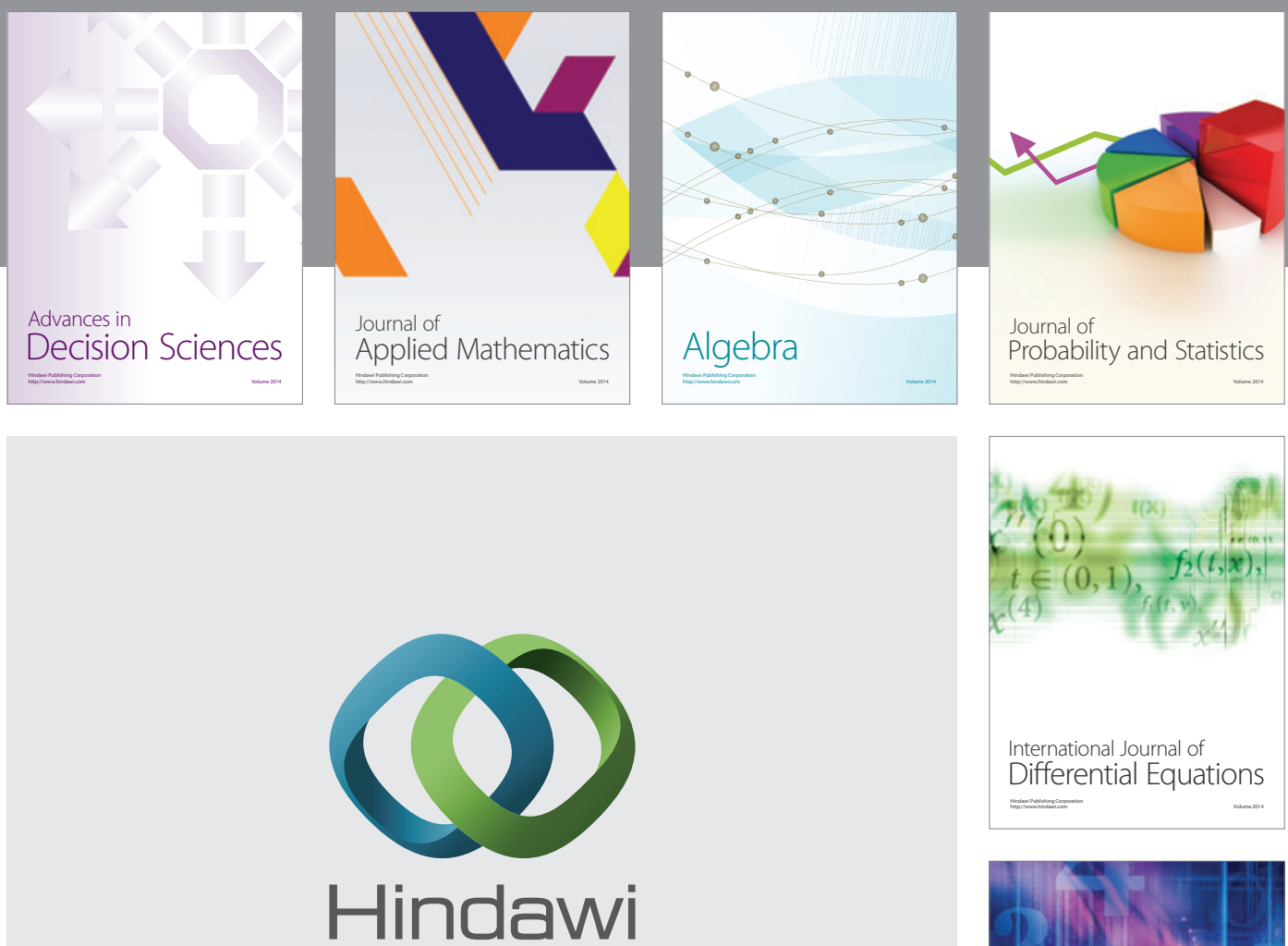

Submit your manuscripts at http://www.hindawi.com
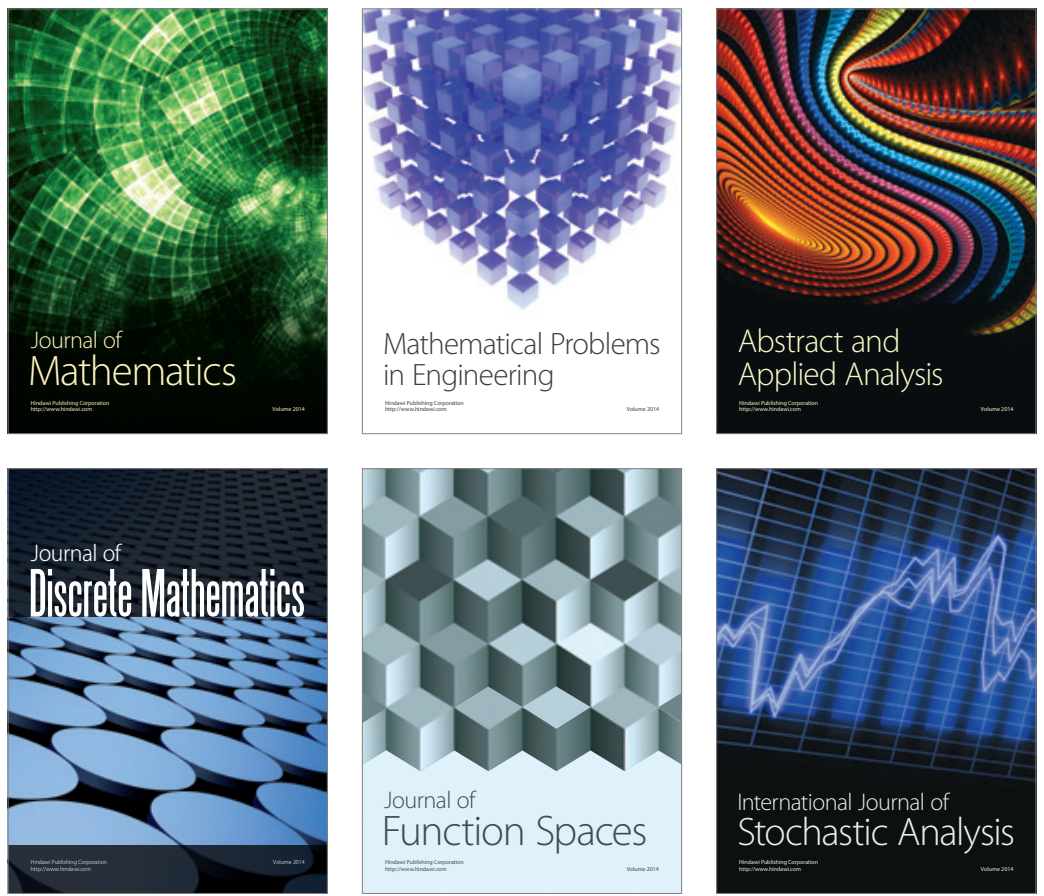

Journal of

Function Spaces

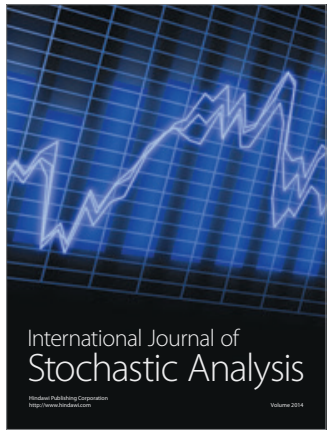

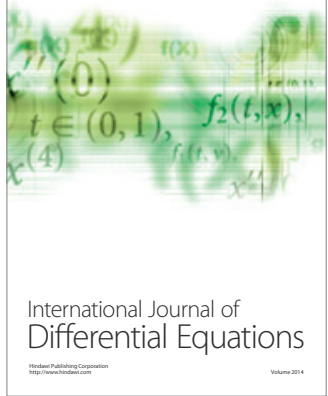
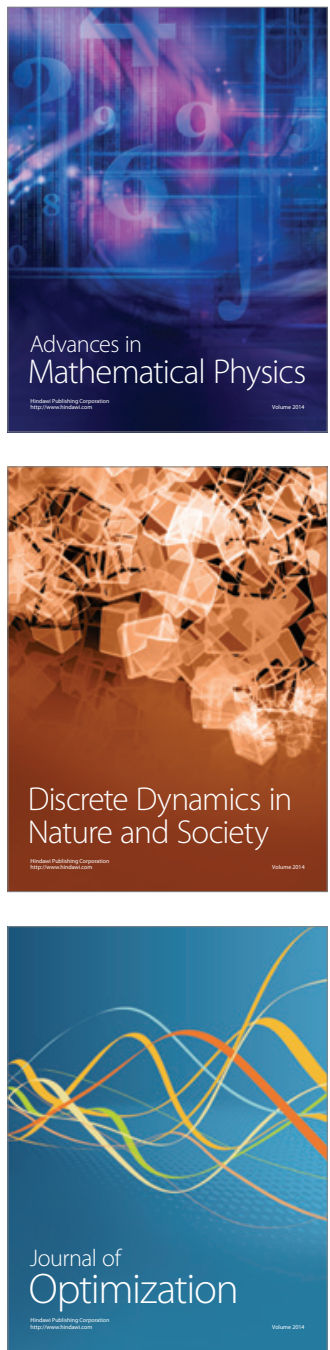